\title{
Peroral Endoscopic Myotomy (POEM) for Complex Achalasia: The Only Realistic Long-Term Treatment
}

\author{
Nikolas Eleftheriadis* \\ Scientific Associate, Endoscopy Department, Metropolitan Hospital Athens, Greece \\ *Corresponding Author: Nikolas Eleftheriadis, Scientific Associate, Endoscopy Department, Metropolitan Hospital Athens, Greece.
}

Received: August 21, 2019; Published: September 27, 2019

DOI: $10.31080 /$ ASGIS.2019.02.0084

Keywords: Complex Achalasia, Heller Myotomy; Per-Oral Endoscopic Myotomy (POEM); Submucosal Endoscopy

POEM has been established as the most modern, innovative, minimal invasive, long-term treatment for all types of esophageal achalasia, with superior characteristics in terms of safety, efficacy and feasibility to all other existing, older and outdated therapies.

POEM approximates the diseased circular esophageal muscle, which is responsible for achalasia, directly and easily through the submucosal tunnel, permitting safe and efficient long myotomy, without damage to other, irrelevant to achalasia, surrounding structures. So, during POEM the operator can clearly identify and cut the muscle fibers, permitting efficient selective circular or fully thickness myotomy, according to his desire, reducing at the same time the risk of complications. Anti-reflux procedure also is not absolutely necessary after POEM, because the normal antireflux mechanisms (diafragmatic crue and angle of His) remain intact due to internal access. This is of great importance particularly in complex achalasia cases, because additional antireflux procedure, could increase operation time and risk of complications, while makes POEM more invasive. In contrary in surgical or LHM myotomy, the normal antireflyx mechanisms were disrupted due to external access, and so antireflux procedures are obligatory, which also increase the risk of complications.

A major safety issue emerged, exclusively by POEM, is the ability to perform myotomy far from the mucosa, inside the submucosal tunnel, the so-called $<<$ third space $>>$, which reduces the risk of serious complications, such as mucosal incisions. During surgical Heller myotomy however, from outside to inside, there is no clear distance between muscle layer and mucosa and risk of serious complications such as incomplete myotomy or mucosal perforation during Heller are more possible.

POEM, however, is not only a minimal invasive, scarless, painless and bloodless procedure, being performed also on endoscopy Department, with short hospitalization and recovery time, alternatively to other existing therapies. POEM permits long myotomy length (up to $25 \mathrm{~cm}$ ), which is absolutely necessary, for types II or III achalasia (Chicago classification), or other esophageal motility disorders, which is practically impossible with any other method. On the other hand, the oldest (100-years-old) surgical, laparoscopic or even robotic Heller myotomy, are still so painfull, aggressive and invasive procedures, because they require both thoracotomy and abdominal incision to approach the esophagus, and to perform as long myotomy as POEM, that practically make them prohibitive in terms of risk and in the era of POEM.

With increase in experience, POEM has been extended and applied to more complex achalasia cases, particularly for sigmoid type achalasia with megaesophagus, after failure of all other existing treatment options, such as after failed surgical or LHM Heller, while it can be applied to elderly or octogenarians or even debilitating patients, with poor general condition and high risk for surgery.

Sigmoid achalasia, particularly sigmoid type II with megaesophagus, as well as failure of previous surgical myotomy, makes further treatment options difficult, while there is no international consensus for the therapy of choice in these complex and severe achalasia cases. Esophagectomy or esophago-jejunal anastomosis could be proposed as the ultimate solution, however with high failure, morbidity and mortality rates.

POEM has the advantage of posterior approach to approximate the diseased circular esophageal muscle, permitting efficient myotomy, far from the scar from previous anterior surgical myotomy and sutures from previous gastropexy. It is worthy to notice that previous surgical Heller myotomy, not only made POEM techically more difficult, but increase the risk of severe post POEM complications. Redo-POEM either posteriorly or anteriorly is also feasible and safe, in short time distance and significantly effective after failed previous anterior or posterior POEM.

In such severe and complex achalasia cases, POEM is not simply an alternative to other existing therapies, but to our opinion, is the only realistic therapeutic option, provided long-term solution, with minimal invasivaness, in cases where all other existing therapies failed, contraindicated or were unrealistic. Obviously, POEM can 
be also easily, safely and effectively applied to all achalasia cases, also to easy, initial stages. Substantially POEM has been established worldwide, currently there are no restriction on the application of POEM in specialized centers, and appears to have replaced all prior treatments of achalasia.

\section{Volume 2 Issue 8 October 2019}

(C) All rights are reserved by Nikolas Eleftheriadis. 